\title{
THE INFLUENCE OF ANNEALING TEMPERATURE ON MAGNETIC PROPERTIES OF VITROVAC 6030
}

\author{
H.K. LaChowicz ${ }^{a, b}$, F. Poplawsik ${ }^{a, b}$, R. ŻUBereK ${ }^{a}$, T. KULIK ${ }^{c}$,

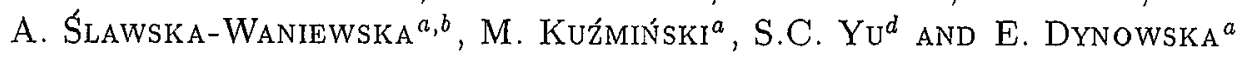 \\ anstitute of Physics, Polish Academy of Sciences \\ Al. Lotników 32/46, 02-668 Warszawa, Poland \\ ${ }^{b}$ College of Science, Al. Lotników 32/46, 02-668 Warszawa, Poland \\ ${ }^{c}$ Department of Materials Science \& Engineering, Warsaw University of Technology \\ Narbutta 85, 02-524 Warszawa, Poland \\ ${ }^{d}$ Department of Physics, Chungbuk National University \\ Cheongju, 360-763, Korea
}

(Received March 2, 1999; revised version April 8, 1999)

\begin{abstract}
Co-Fe-Mo-Mn-Si-B metallic glass ribbon (Vitrovac 6030) was subjected to the isothermal annealing at temperatures in the range $523-873 \mathrm{~K}$ so as to produce a series of samples with gradually coarser microstructure. For this series of samples a giant increase in the coercivity, exceeding three orders of magnitude, is observed. This increase is interpreted in terms of the strengthening of the pinning effect of the nanocrystalline structure on the moving domain walls. It is shown that the anisotropy of the created crystallites is mainly responsible for the increase in the pinning force since the effective anisotropy seen by the wall becomes larger and larger with the gradual increase in the density and size of the grown particles.
\end{abstract}

PACS numbers: $75.50 . \mathrm{Cc}, 75.50 . \mathrm{Kj}, 75.60 . \mathrm{Ch}$

\section{Introduction}

As commonly known, coercivity of magnetic materials strongly depends on their microstructure, mainly because of the interaction of the domain walls with defects which are generally identified with grains of which a given polycrystalline magnet is composed. Therefore, the average size of defects pinning the wall movement scales linearly with the mean grain size. As it has been shown, both theoretically and in the numerous experiments, coercivity scales with the mean grain (defect) size. However, this scaling. proceeds differently depending on the value of the mean grain size $D$ compared with the domain wall width $\delta$, the quantity 
which can be identified with the correlation length for ferromagnetic exchange interaction. In the regime where grains are larger than the wall width, $D>\delta$, coercivity decreases with an increase in the grain size according to the well known 1/D-dependence (see, e.g. [1]).

However, the invention of nanocrystalline magnets [2], produced by well controlled annealing of metallic glasses and known under their trade name FINEMET, has shown that coercivity of these materials scales proportionally to $D^{6}$ (see, e.g. [3]). Hence, coercivity rapidly increases with an increase in the grain size contrary to the case when $D>\delta$. In the nanocrystalline magnets, the mean size of grains, which usually is of the order of $10-30 \mathrm{~nm}$, is smaller than the wall width, $D<\delta$. Because of this relation, the basic mechanism for the coercivity in the considered nanocrystalline magnets is that the local magnetocrystalline anisotiopy of the individual grains is largely averaged out due to ferromagnetic exchange interaction acting between magnetically coupled grains. However, both scaling relations described above, are correct only for materials in which the volume density of crystalline phase is high enough so as the exchange interaction can be effective.

Therefore, it would be interesting to recognize the evolution of magnetic properties (coercivity, in particular) of magnets obtained by successive devitrification of metallic glass in which created nanograins are much smaller than the wall width and in which their volume density is low enough, so as they are not directly coupled magnetically.

Though the studies on the influence of the progressive devitrification of metallic glasses on their coercivity have appeared in the literature (see, e.g. [4-6]), nevertheless, the experimental methods used in the cited works as well as the interpretation of the reported results differ in substance from those used in the present work.

\section{Experimental}

For the experiment Co-based metallic glass (Vitrovac 6030 of the nominal composition $\mathrm{C}_{71} \mathrm{Fe}_{1} \mathrm{Mo}_{1} \mathrm{Mn}_{4} \mathrm{Si}_{14} \mathrm{~B}_{9}$, produced by Vacuumschmelze, GmbH, Hanau, Germany) was chosen as the parent material for partially devitrified samples. The main motive for such a selection was that this matierial in its as-quenched state exhibits negligible magnetostriction $\left(<0.2 \times 10^{-6}[7]\right)$, the property which is crucial from the viewpoint of the designed studies. For magnetostrictive material, magnetoelastic long-range interactions could have mainly been responsible for the coercivity, thus preventing to investigate the influence of the nanocrystallites themselves. The samples used for measurements were $25 \mu \mathrm{m}$ thick, $5 \mathrm{~mm}$ wide and $70 \mathrm{~mm}$ long. In order to obtain a series of samples exhibiting various density of the nanocrystalline phase, they were isothermally annealed for $1 \mathrm{~h}$ at the temperatures ranging from 523 up to $873 \mathrm{~K}$. All samples were heat treated in the evacuated quartz ampoules to prevent surface oxidation (it was shown that annealing in air, even at the temperature as low as $523 \mathrm{~K}$, leads to the formation of surface oxides, non-magnetic borosilicate [8]). Prior to annealing, a differential scanning calorimetry (DSC) at the heating rate $10 \mathrm{~K} /$ min (Perkin-Elmer DSC-7) was used to determine the range of the crystallization temperatures of the parent metallic glass. Measurement of the total magnetic moment of the as-quenched 
sample annealed at the same heating rate has also been accomplished applying vibrating sample magnetometer (1.2 T MagLab VSM - Oxford Instruments, Ltd.). These measurements were carried out in order to find out magnetic phases of the products of devitrification comparing both curves. Devitrification processes were followed by X-ray diffraction performed using $\mathrm{Cu} K_{\alpha}$ radiation in order to identify the structure of the nanocrystallites created at the time of annealing.

Coercivity of the samples has been determined measuring at room temperature their saturated hysteresis loops along the length of the sample. These measurements were carried out using conventional inductive method realized by means of a computerized setup operating with the frequency $25 \mathrm{~Hz}$ of the sawtooth shaped magnetizing field. Figure 1 presents a typical hysteresis loop obtained in the experiment.

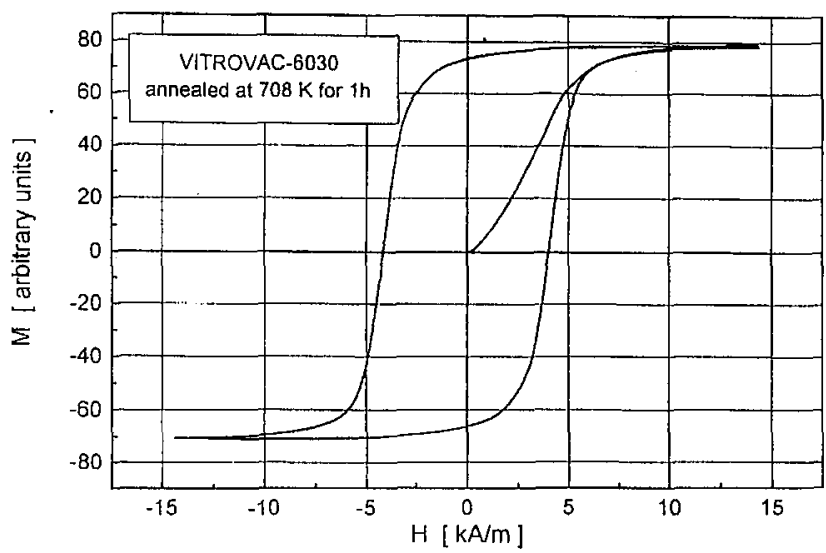

Fig. 1. A typical hysteresis loop along with the virgin magnetization curve is shown (for the sample annealed at $708 \mathrm{~K}$ for $1 \mathrm{~h}$ ).

Since magnetostriction is considered as the crucial property from the viewpoint of the studies carried on, its evaluation with the annealing temperature was also measured. For this purpose, strain modulated ferromagnetic resonance (SMFMR) was utilized using FMR-spectrometer operating at $9.1 \mathrm{GHz}$ and with the field sweep up to $0.4 \mathrm{~T}$. The spectrometer was equipped with the facilities allowing to introduce stress into the sample in a controlled way (the details of the method can be found elsewhere [9]).

Magnetic domain structure, appearing on the surface of the samples, was also observed by means of Kerr-effect technique using a computerized setup in which cancellation of the non-magnetic background is possible. Prior to observations, the samples were demagnetized by an a.c. field.

In order to find the correlation between coercivity and microstructure of the gradually devitrified samples, the latter was inspected by means of the transmission electron microscopy (TEM) using Philips EM-300 microscope operating with $100 \mathrm{keV}$. Samples were thinned down by ion-etching. 


\section{Results and discussion}

Figure 2 shows crystallization characteristics of the used parent metallic glass. While in Fig. 2a the DSC-curve obtained for the as-quenched sample by continuous heating at the rate of $10 \mathrm{~K} / \mathrm{min}$ is presented, Figs. 2ls,c show the evolution of magnetic moment for the sample heated at the same rate. The dependence presented in Fig. $2 b$ reveals temperature changes of the moment measured within the plane of the sample, whereas that in Fig. $2 c$ presents temperature evolution of the perpendicular component of magnetic moment. Both dependencies were measured at the applied field of $0.6 \mathrm{~T}$. As seen in Fig. $2 \mathrm{~b}$ and $\mathrm{c}$, the perpendicular component does not exceed $1 \%$ of that measured within the plane, the result not surprising because of the existing strong demagnetizing field above the surface of the sample. The dependence obtained for the heated up sample (Fig. 2b) shows that the saturation polarization, $J_{\mathrm{s}}$, equals $0.83 \mathrm{~T}$ (the specific density of the sample is equal to $7.79 \mathrm{~g} / \mathrm{cm}^{3}$ [7]) and the estimated Curie point equals around $660 \mathrm{~K}$, being in a good agreement with the values given by the producer $(0.82 \mathrm{~T}$ and $638 \mathrm{~K}$ [7], respectively). It is, however, worth noticing that both dependencies, thermal and magnetic (see Figs. $2 a$ and b), indicate very good agreement regard-

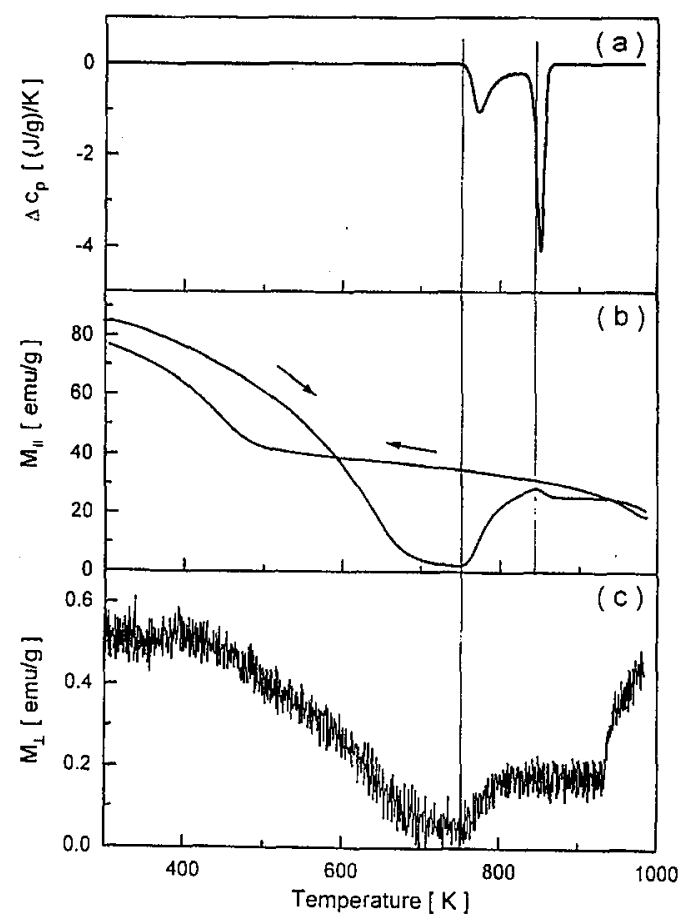

Fig. 2. Crystallization characteristics of the parent Co-Fe-Mo-Mn-Si-B metallic glass: DSC scan taken at the heating rate of $10 \mathrm{~K} / \mathrm{min}$ (a); VSM scan at the same rate of heating displaying the in-plane component of magnetization (b); the same as (b) but for the perpendicular component of magnetization (c). 
ing the crystallization processes. The DSC-curve indicates that crystallization of the parent glass proceeds in two well separated steps and that both created phases exhibit magnetic behavior, as it is revealed by VSM-measurements (Fig. 2b). The onset of crystallization takes place around $750 \mathrm{~K}$, whereas the second step of this process is initiated at about $830 \mathrm{~K}$. X-ray diffraction patterns obtained for partially crystallized samples are quite complex (see Fig. 3 which presents such a pattern for the sample annealed at $773 \mathrm{~K}$ ). An analysis of the patterns obtained for all partially crystallized samples shows that the created particles most probably comprise of $\mathrm{CO}_{3} \mathrm{~B}$ crystalline phase mixed with a very small amount of $\mathrm{Co}_{2} \mathrm{Mo}_{3}$ phase (the parent amorphous alloy contains only 1 at.\% of Mo). The Curie temperature of $\mathrm{Co}_{3} \mathrm{~B}$ equals $760 \mathrm{~K}[10]$. However, the value of this temperature for the crystalline phase grown within the first crystallization step at the time of continuous heating of the parent material seems to be much higher than that for $\mathrm{Co}_{3} \mathrm{~B}$ (see Fig. 2b). It suggests that the composition of the crystallites is more complex than that determined from the $\mathrm{X}$-ray diffraction patterns.

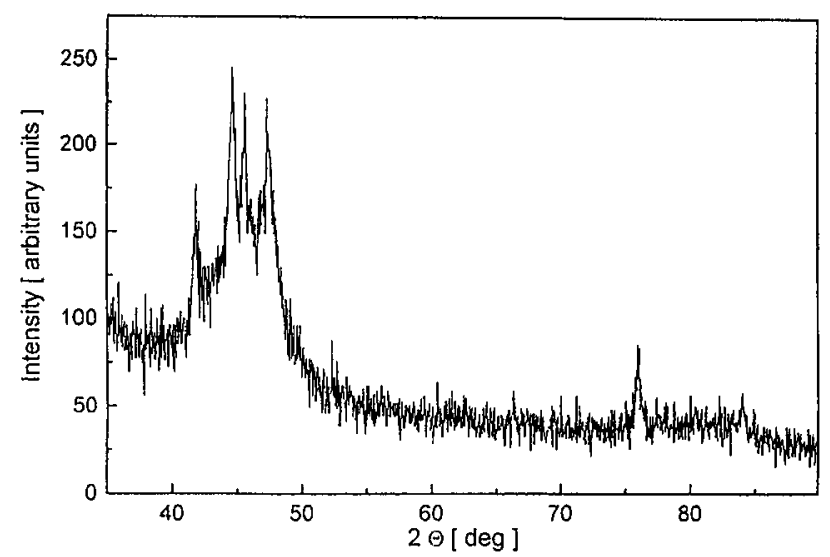

Fig. 3. X-ray diffraction spectrum for the sample annealed at $753 \mathrm{~K}$ for $1 \mathrm{~h}$.

Heating the sample at the temperatures higher than the onset of the secand crystallization step leads to the total crystallization of the sample. The above anticipations were confirmed by transmission electron microscopy (TEM) observations of the microstructure of the samples annealed isothermally for 1 hour at various temperatures. For the temperatures in the range within the first crystallization peak, tiny crystallites are grown the size of which and their volume density become the larger the higher the annealing temperature. This effect is seen in Fig. 4 which presents TEM micrographs of the structure of the samples annealed at progressively increasing temperature. For the temperatures above the second peak, the sample becomes totally crystallized as seen in Fig. 4d.

Figure 5 presents the dependence of the coercivity (measured at room temperature) on the annealing temperature in the range from $523 \mathrm{~K}$ up to $873 \mathrm{~K}$, obtained for a series of samples isothermally heated for 1 hour at successively in- 

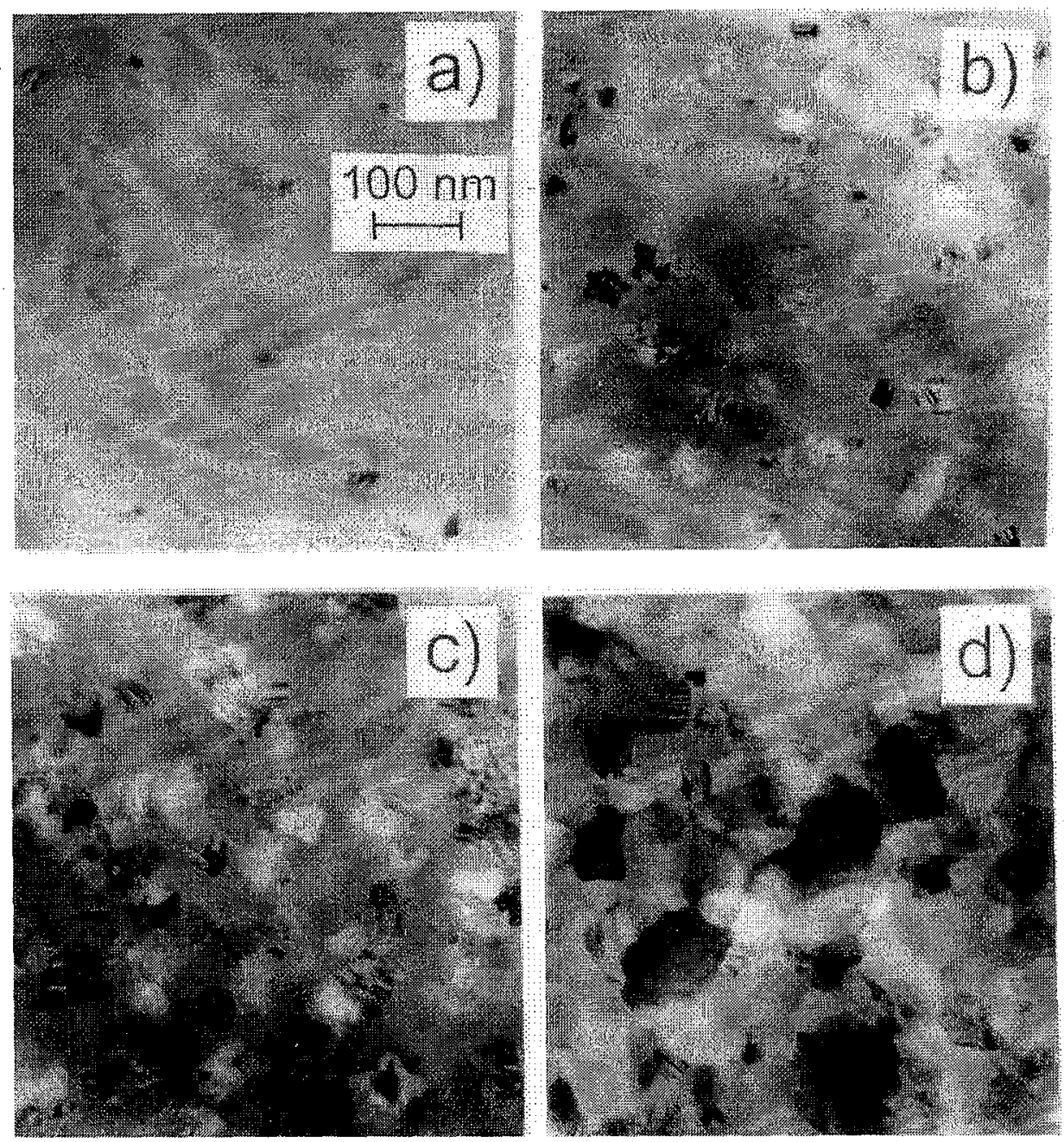

Fig. 4. TEM micrographs obtained for the samples: annealed at $693 \mathrm{~K}$ (a), at $708 \mathrm{~K}$ (b), at $753 \mathrm{~K}(\mathrm{c})$, and at $873 \mathrm{~K}(\mathrm{~d})$. Each sample was annealed for $1 \mathrm{~h}$. The magnification is the same for all the micrographs.

creasing temperature. As it is seen in this figure, the change of coercivity exceeds three orders of magnitude, achieving its "saturation" value of about $32 \mathrm{kA} / \mathrm{m}$ for the sample annealed at $873 \mathrm{~K}$. Surprisingly, the lowest value of this parameter, equal to $7 \mathrm{~A} / \mathrm{m}$, was obtained for the as-quenched sample on contrary to the expectation that annealing at relatively low temperature usually leads to a decrease in coercivity owing to stress relaxation. The above result can, however, be explained considering the fact that the parent metallic glass exhibits extremely low magnetostriction $\left(\approx 10^{-7}\right.$, as measured using SMFMR method, see Fig. 6a) and, therefore, magnetoelastic contribution to the coercivity is in this case negligible. 


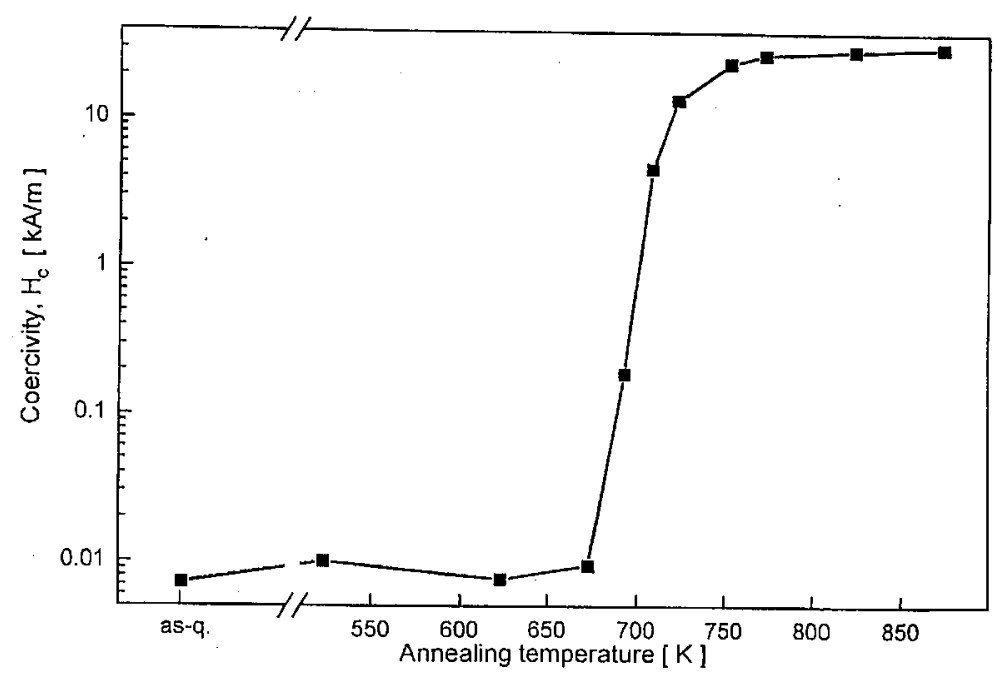

Fig. 5. Dependence of the coercivity on isothermal annealing temperature for a series of Co-Fe-Mo-Mn-Si-B samples measured at room temperature.

As seen in Fig. 5, the dominant change of coercivity occurs in a relatively narrow range of the annealing temperatures (from 632 up to $732 \mathrm{~K}$ ). This fact becomes comprehensible if one considers TEM micrographs presented in Fig. 4 which shows that the dominant changes of the microstructure take also place for the samples annealed in the same range of temperatures. In this range, initially nucleated tiny crystallites (Fig. 4a) grow up with an increase in the temperature of annealing, occupying more and more of the whole volume (Figs. $4 \mathrm{~b}$ and c) as long as the total crystallization is completed (Fig. 4d). Further increase in the annealing temperature generates only small changes of the coercivity.

However, the rapid rise of coercivity starts at much lower temperature of annealing than that disclosed by DSC and magnetic measurements for the onset of the primary crystallization (as seen comparing Fig. 2 and Fig. 5). Two reasons can cause this effect. First is that the crystallization temperature is a dynamic quantity which is the higher the larger is the rate of rise of the heating temperature (the samples are annealed at the isothermal conditions). Secondly, the crystallites can be nucleated at much lower temperature than that at which the onset of crystallization is revealed and the methods used are not sensitive enough to detect such a small amount of the crystalline phase. On the other hand, coercivity is extremely sensitive to even the smallest "defects" which may act as the pinning centers.

For a magnet build up from an amorphous medium containing non-directly-interacting randomly distributed nanocrystallites, Porteseil and Goeffroy have shown that its coercivity can be estimated from simple statistical arguments [11]. Applying these arguments, they have derived the expression which describes the influence of the domain wall pinning on the coercivity of the material. The effect of pinning arises from the anisotropy energy of the crystallites present within the volume of the moving wall. This expression has the form 


$$
H_{\mathrm{c}}=n^{1 / 2} \delta^{-3 / 2} K_{1} v / 12 J_{\mathrm{s}}
$$

where $n$ is the volume density of the nanocrystallites, $\delta$ - the wall width, $K_{1}$ the anisotropy constant of the crystallite, $v$ - its mean volume, and $J_{s}$ - the saturation polarization of the sample.

It can easily be noticed from the albove expression that coercivity should rise with an increase in the density and size of the crystallites, assuming that the anisotropy. of the crystallites does not vary and that the wall width is mainly determined by the properties of the amorphous matrix and, therefore, does not change significantly.

Assuming a hypothetical model material in which only the size of the crystallites could independently be controlled, then according to Eq. (1) the coercivity should scale with $D^{3}$ - the scaling relation which differs from those mentioned previously. Unfortunately, such a material does not exist in practice and for a real nanocrystalline magnet a change of the crystallite size is always followed by variations of all parameters occurring in Eq. (1).

The samples studied fulfil the assumptions under which Eq. (1) was derived, therefore, this expression can be used to interpret the observed changes of coercivity.

Performed calculation for the sample annealed at $693 \mathrm{~K}$ shows that the value of coercivity obtained from Eq. (1) is more than two orders of magnitude smaller than that obtained experimentally. It was assumed in this calculation that the anisotropy energy of the crystallites is the same as that for pure cobalt $\left(K_{1}=10^{5} \mathrm{~J} / \mathrm{m}^{3}\right)$ and that the wall width equals $\delta=10^{-6} \mathrm{~m}$ - a. typical value for metallic glasses (see, e.g. [12]). The density and mean volume of the crystallites (assuming their spherical shape) were estimated from TEM micrographs and are equal to $n \approx 1.4 \times 10^{21} \mathrm{~m}^{-3}$ and $v=4 \times 10^{-24} \mathrm{~m}^{3}$, respectively. The measured value of $0.83 \mathrm{~T}$ for saturation polarization was used in this calculation.

The observed large discrepancy between the calculated and experimental values of coercivity may result from the fact that some of the values of the quantities occurring in Eq. (1) were chosen arbitrarily, the anisotropy energy and, in particular, domain wall width (reducing the assumed value of the latter quantity by an order of magnitude gives the value of the coercivity of the right order of magnitude). Additionally, the accuracy of the estimation of the volume density and mean volume of the crystallites, performed using TEM micrographs, is naturally not very high.

Equation (1) can, however, be used to interpret the experimental results in a different way, viz. to compare the dynamics of an increase in coercivity with the annealing temperature, observed in the experiment with that calculated from this equation. Assuming that the anisotropy, wall width and saturation polarization do not change significantly with the temperature of annealing, then, according to Eq. (1), coercivity should scale with the product $n^{1 / 2} \times v$ (its values, estimated for the samples amnealed at 693,708 , and $735 \mathrm{~K}$, are given in Table). Two last columns in this table display the introduced parameters $k_{\exp }$ and $k_{\text {cal }}$, equal to the values of the experimental and calculated coercivity, respectively, both normalized in relation to the values of coercivity for the sample annealed at $693 \mathrm{~K}$. 
TABLE

Dynamics of the variation of coercivity.

\begin{tabular}{c|c|c|c|c|c}
\hline \hline $\begin{array}{c}T_{\text {ann }} \\
{[\mathrm{K}]}\end{array}$ & $\begin{array}{c}D \\
{[\mathrm{~nm}]}\end{array}$ & $\begin{array}{c}n \times 10^{-21} \\
{\left[\mathrm{~m}^{-3}\right]}\end{array}$ & $\begin{array}{c}n^{1 / 2} v \times 10^{14} \\
{\left[\mathrm{~m}^{-1 / 2}\right]}\end{array}$ & $k_{\exp }$ & $k_{\text {cal }}$ \\
\hline 693 & 20 & 1.38 & 15.5 & 1 & 1 \\
708 & 50 & 2.33 & 315.7 & 25.0 & 20.4 \\
753 & 70 & 1.58 & 710.6 & 131.0 & 45.8
\end{tabular}

Both parameters give a measure of the dynamics of the rise of coercivity with the amnealing temperature. As seen in the columns mentioned above, the agreement between calculated and experimental values of the introduced parameters is much better than that for the absolute values of the coercivity obtained previously.

The discrepancies between the values of both parameters increase with the temperature of annealing (see Table). Since the volume content of the crystalline phase increases with the rise of this temperature, the effective anisotropy seen by the wall becomes larger and larger, and, consequently, its width decreases inconsistently with the earlier assumption that the wall width does not change at these conditions. This effect which is, however, difficult to be controlled is the most probable source of the observed large discrepancies when using Eq. (1) to interpret the experimental results.

Figure 6 shows the dependencies of magnetostriction (measured at room temperature) as a function of the annealing temperature. The plotted quantity was calculated from the data obtained by means of the SMFMR method, assuming the values of the $g$-factor and of the Young modulus equal 2.2 and $220 \mathrm{GPa}$, respectively (a typical value for metallic glasses). As seen in this figure, magnetostriction

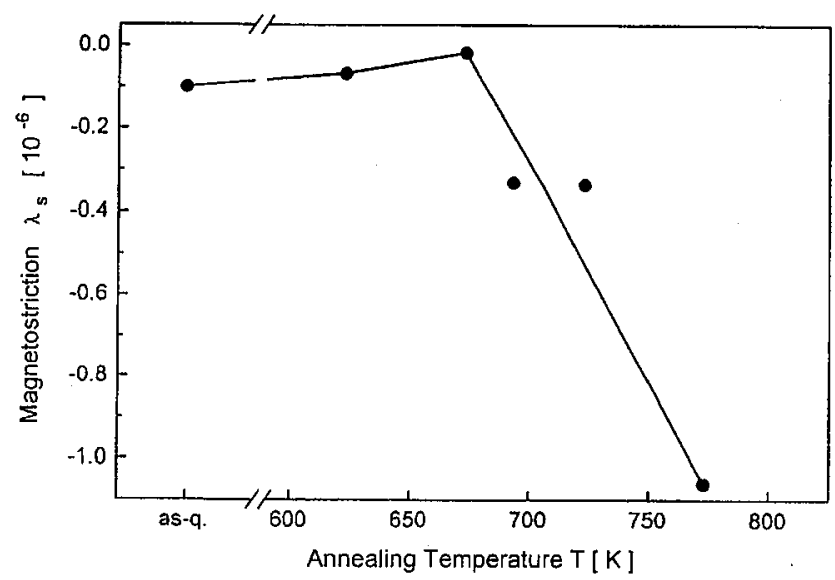

Fig. 6. Dependence of the magnetostriction on annealing temperature for a series of samples (calculated from the data obtained at room temperature using SMFMR technique). 
displays extremely low values $\left(\approx 10^{-7}\right)$ for the samples annealed in the range of temperatures lower than that at which the onset of crystallization occurs. In the range of higher annealing temperatures, within which primary crystallization takes place, magnetostriction rises almost linearly in its absolute value becoming more and more negative, achieving the value $10^{-6}$ for the sample heated at $773 \mathrm{~K}$. The observed very low magnetostriction confirms the anticipation made earlier that magnetoelastic interactions have negligible influence on coercivity. This conclusion is additionally confirmed by observation of domain structure in the as-quenched sample (see Fig. 7a) which does not display complex and irregular pattern typical of magnetostrictive samples in which magnetoelastic anisotropy due to the quenched-in stresses plays a significant role. The observed domain pattern for this sample in its demagnetized state is as simple as possible, consisting of two domains separated by the straight wall aligned with the length of the ribbon sample. For
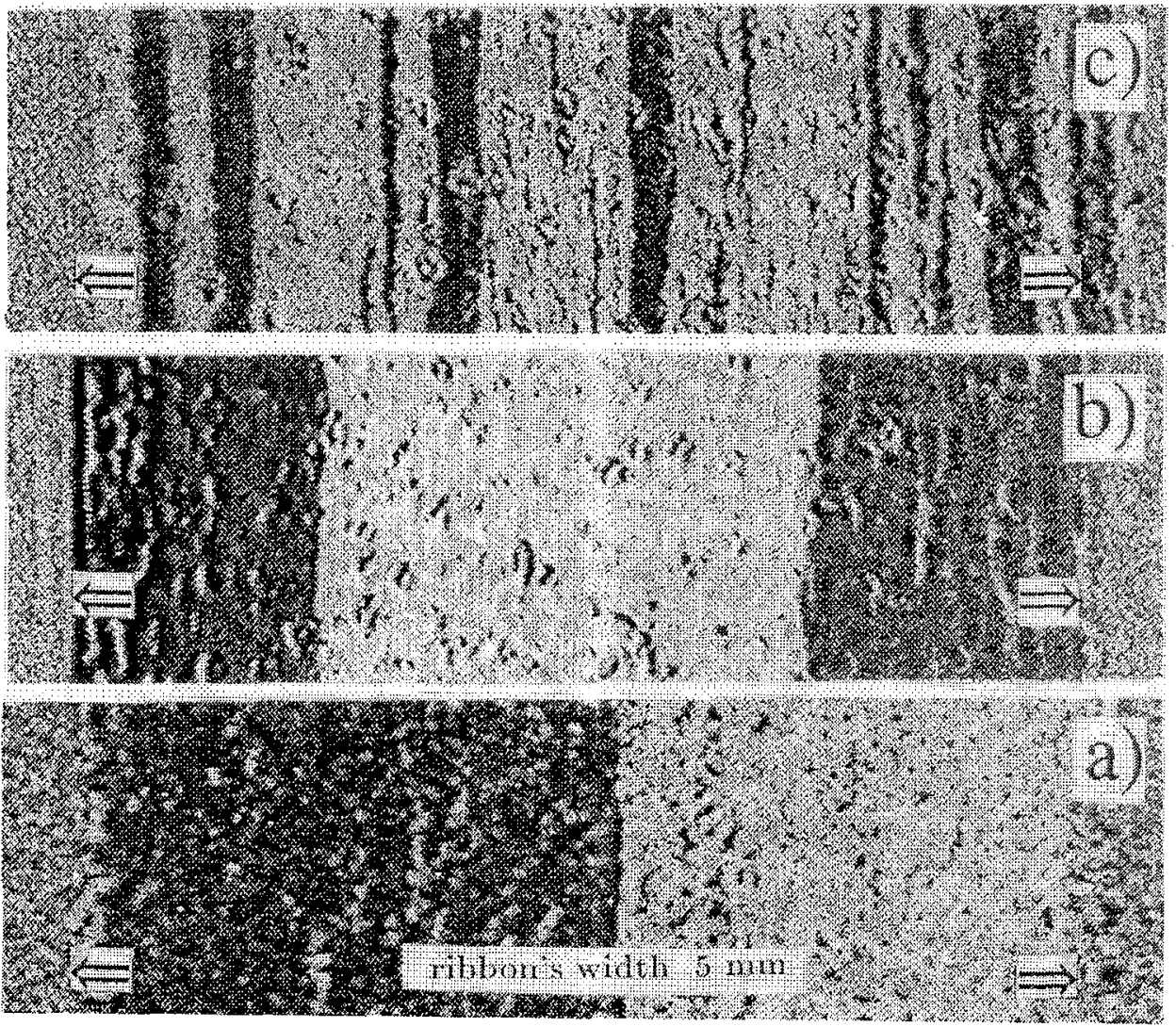

Fig. 7. Kerr-effect images of domain structure observed for samples: in as-quenched state (a), annealed at $673 \mathrm{~K}(\mathrm{~b})$, and at $693 \mathrm{~K}$ (c). The patterns (b) and (c) were taken after demagnetization of the samples by an a.c. field. 
the samples annealed at the temperature at which the crystallites appear, their domain patterns after demagnetization consist of several longitudinal domains, the number of which increases with the rise of the heating temperature (see Fig. 7b and c). It is not surprising since the force pinning the walls becomes stronger the larger volume density of the crystallites is.

As claimed in [13], at the early stages of crystallization of CoFe-based metallic glasses, iron-rich crystal-like clusters are formed which act as nucleation sites in the glass to crystal transformation. Thus, the growing crystallites may contain relatively large amount of iron (the analysis of the $\mathrm{X}$-ray diffraction patterns does not reveal this effect) causing that the amorphous matrix becomes poorer of this element and, consequently, magnetostriction grows more negative. The same effect has been observed for gradually devitrified CoFe-based amorphous alloy [14] but of different composition than that used in the present work.

For still higher temperatures, in the range which corresponds with the second step of crystallization, the observed resonance spectra are so smeared that calculation of magnetostriction becomes quite unreliable.

\section{Conclusions}

It is shown in the present paper that; progressive devitrification of Co-based metallic glass (Vitrovac 6030) leads to an increase in the coercivity by more than three orders of magnitude. The mechanism mainly responsible for this giant increase is the effect of pinning on the moving domain walls which originates from the created crystallites of the size smaller than the correlation length for ferromagnetic exchange interaction. Their size, volume density and magnetocrystalline anisotropy are the crucial factors determining the coercivity of the devitrified metallic glass. The results presented also show a possibility of tailoring the nanocrystalline magnets according to the requirements for a given application.

It is worth noticing that the series of samples used in the reported experiment proved to be an excellent model material to verify Néel's theory of the well known Lord Rayleigh empirical rule which describes the initial part of the virgin magnetization curve [15].

\section{Acknowledgments}

The work was supported in part by the Committee for Scientific Research (Poland) under research grant No. 8 T11B 048 10. The authors are grateful to Dr. G. Herzer (Vacuumschmelze, GmbH, Hanau, Germany) for supplying metallic glass ribbon used for the experiments.

\section{References}

[1] F. Pfeifer, C. Radeloff, J. Magn. Magn. Mater. 19, 190 (1980).

[2] Y. Yoshizawa, S. Oguma, K. Yamauchi, J. Appl. Phys. 64, 6044 (1988).

[3] G. Herzer, NanoStructured Mater. 1, 263 (1992).

[4] R.C. O'Handley, J. Megusar, S-W. Sun, Y. Hara, N.J. Grant, J. Appl. Phys. 57, 3563 (1985).

[5] G. Bottoni, D. Gandolfo, A. Cecchetti, J. Appl. Phys. 79, 5469 (1996). 
[6] C. Gómez-Polo, D. Holzer, M. Multigner, E. Navarro, P. Agudo, A. Hernando, M. Vázquez, H. Sassik, R. Grössinger, Phys. Rev. B 53, 3392 (1996).

[7] Amorphous Metals VITROVAC, Catalogue, Edition 7/89, Vacuumschmelze GmbH, Hanan, Germany.

[8] C.K. Kim, R.C. O'Handley, Mater. Sci. Eng. B 38, 16 (1996).

[9] R. Żuberek, H. Szymczak, G. Suran, K. Ounadjela, Solid State Films 188, 1 (1990).

[10] T. Shinohara, H. Watanabe, J. Phys. Soc. Jpn. 20, 2020 (1965).

[11] J.L. Porteseil, O. Goeffroy, J. Magn. Magn. Mater. 140-144, 1855 (1995).

[12] H. Kronmüller, M. Fähnle, M. Domann, H. Grim, M. Grim, B. Gröger, J. Magn. Magn. Mater. 13, 53 (1979).

[13] A. Serebryakov, L. Voropayeva, Yu. Jevin, N. Novokhatskaya, G. Abrosimova, NanoStructured Mater. 4, 851 (1994).

[14] H.K. Lachowicz, T. Kulik, R. Żuberek, L. Małkiński, M. Kuźmiński, A. Ślawska-Waniewska, J.S. Munoz, J. Magn. Magn. Mater. 190, 267 (1998).

[15] H.K. Lachowicz, F. Popławski. S.N. Kane, M. Kuźmiński, T. Kulik, Acta Phys. Pol. A 95, 287 (1999). 